\title{
Calculating topological indices of certain OTIS interconnection networks
}

https://doi.org/10.1515/chem-2019-0029

Received Apr 21, 2018; accepted Oct 10, 2018

\begin{abstract}
Recently, increasing attention has been paid to The Optical Transpose Interconnection System (OTIS) network because of its prospective applications in architectures for parallel as well as distributed systems [27, 28]. Different interconnection networks in the context of topological indices are researched recently in $[25,26]$. This article includes the computions of the general Randi'c, first and second Zagreb, general sum connectivity, first and second multiple zagreb, hyper zagreb, $A B C$ and $G A$ indices for OTIS (swapped and biswapped) networks by taking path and $k$-regular graph on $n$ vertices as a base graphs. In addition, some delicated formulas are also obtained for the $A B C_{4}$ and $G A_{5}$ indices for the OTIS biswapped networks by considering basis graph as a path and $k$-regular graph of order $n$.
\end{abstract}

Keywords: Optical Transpose Interconnection System (OTIS) network, OTIS swapped networks, OTIS biswapped networks, molecular topological indices

\section{Introduction and Preliminaries}

As a new emerging science, Cheminformatics is related to chemistry, mathematics and computer sciences, whose major components include Quantitative structure-activity (QSAR) and structure-property relationships (QSPR) and the components can contribute to the research on physicochemical properties of chemical compounds.

\footnotetext{
^Corresponding Author: Wei Gao: School of Information Science and Technology, Yunnan Normal University, Kunming, 650500, China; Email: gaowei@ynnu.edu.cn

Adnan Aslam: University of Engineering and Technology, Lahore(RCET), Pakistan; Email: adnanaslam15@yahoo.com Safyan Ahmad: Abdus Salam School of Mathematical Sciences GC University, Lahore, Pakistan; Email: safyank@gmail.com Muhammad Ahsan Binyamin: Department of Mathematics, GC University, Faisalabad, Pakistan;

Email: ahsanbanyamin@gmail.com
}

əopen Access. ( 2019 A. Aslam et al., published by De Gruyter. (cc) BY License
As a numeric quantity, a topological index is closely related to a graph which is invariant under graph automorphism and can characterize the topology of a graph. Numerous applications of graph theory can be found in structural chemistry. Its first well-known application in chemistry was the study of paraffin boiling points by Wiener([35]). Various topological indices were introduced following this study that explained physico-chemical properties.

Originally, the main function of optical transpose interconnection system (OTIS) networks was to offer efficient connectivity for new optoelectronic computer architectures which benefit from both optical and electronic technologies [24]. In OTIS networks, processors are organized into clusters. Electronic interconnects are utilised between processors within the same cluster, while optical links are utilised for intercluster communication. Numerous algorithms have been devised for routing, selection/sorting $[13,16,30,31]$, certain numerical computations [29], Fourier transform [7], matrix multiplication [15], and image processing [14].

The structure of an interconnection network can be mathematically modeled by a graph. The vertices of this graph represent the processor nodes and the edges represent the links between the processors. The topology of a graph determines the way in which vertices are connected by edges. From the topology of a network, certain properties can easily be determined. The diameter is determined as the maximum distance between any two nodes in the network. The number of links connected to a node determines the degree of that node. If this number is the same for all nodes in the network, the network is called regular.

In this article, $G$ is considered to be a simple graph with the vertex set $V(G)$ and the edge set $E(G)$. Since $u \in$ $V(G)$, we denote $N_{u}$ as the set of its neighbors in $G$, the degree $d_{u}$ of vertex $u$ is $d_{u}=\left|N_{u}\right|$ and $S_{u}=\sum_{v \in N_{u}} d_{v}$. The first degree based topological index is Randić index brought about by Milan Randić [32] in 1975. The general Randić connectivity index [9] of $G$ is denoted as

$$
R_{\alpha}(G)=\sum_{u v \in E(G)}\left(d_{u} d_{v}\right)^{\alpha},
$$


where $\alpha$ is a real number. When $\alpha=-\frac{1}{2}$, then $R_{-1 / 2}(G)$ is known as Randić connectivity index of $G$.

The sum-connectivity index was introduced by $B o$ Zhou and Nenad Trinajstić [10] to be described as

$$
\operatorname{SCI}(G)=\sum_{u v \in E(G)} \frac{1}{\sqrt{d_{u}+d_{v}}} .
$$

The general sum-connectivity index $\chi_{\alpha}(G)$ was proposed in 2010 in [11]:

$$
\chi_{\alpha}(G)=\sum_{u v \in E(G)}\left(d_{u}+d_{v}\right)^{\alpha} .
$$

Approximate four decades ago, Ivan Gutman and Trinajstić proposed an significant topological index. To be simple, first Zagreb index denoted by $M_{1}(G)$ was denoted as the sum of degrees of end vertices of all edges of $G$. We describe the first Zagerb $M_{1}(G)$ and second Zagreb $M_{2}(G)$ is defined as

$$
\begin{gathered}
M_{1}(G)=\sum_{v \in V(G)} d_{v}^{2}=\sum_{u v \in E(G)}\left[d_{u}+d_{v}\right] \\
M_{2}(G)=\sum_{u v \in E(G)}\left[d_{u} \times d_{v}\right]
\end{gathered}
$$

where $d(u)$ is the degree of the vertex $u$ in the graph $G$.

Ghorbani et al. [21] described two new versions of the Zagreb indices of a graph $G$ in 2012 to be the first multiple Zagreb index $P M_{1}(G)$, second multiple Zagreb index $\mathrm{PM}_{2}(G)$, which are denoted as

$$
\begin{aligned}
& P M_{1}(G)=\prod_{u v \in E(G)}\left[d_{u}+d_{v}\right] \\
& P M_{2}(G)=\prod_{u v \in E(G)}\left[d_{u} \times d_{v}\right]
\end{aligned}
$$

Currently, Shirdel et al. [33] raised up the hyper-Zagreb index:

$$
H M(G)=\sum_{u v \in E(G)}\left[d_{u}+d_{v}\right]^{2}
$$

Atom-bond connectivity (ABC) index proposed by Estrada et al. in [19] have numerous applications for its connectivity topological index. The $A B C$ index of graph $G$ is defined as

$$
A B C(G)=\sum_{u v \in E(G)} \sqrt{\frac{d_{u}+d_{v}-2}{d_{u} d_{v}}} .
$$

The fourth member of the class of $A B C$ index is $A B C_{4}$ was introduced by $M$. Ghorbani et al. in [22] as:

$$
A B C_{4}(G)=\sum_{u v \in E(G)} \sqrt{\frac{S_{u}+S_{v}-2}{S_{u} S_{v}}} .
$$

D. Vukicevic and B. Furtula raised up the geometric arithmetic (GA) index in [34] and described as

$$
G A(G)=\sum_{u v \in E(G)} \frac{2 \sqrt{d_{u} d_{v}}}{d_{u}+d_{v}} .
$$

Currently, the fifth version of $G A$ index is $\left(G A_{5}\right)$ is put forward by Graovac et al. in [23] as

$$
G A_{5}(G)=\sum_{u v \in E(G)} \frac{2 \sqrt{S_{u} S_{v}}}{S_{u}+S_{v}} .
$$

In 2014, Hayat et al. computed the detailed expressions for the $A B C, G A, A B C_{4}$ and $G A_{5}$ indices for diverse networks like silicates, honeycomb, hexagonal and oxides in [25]. They also studied the general Randić, $A B C, G A$ and first Zagreb indices for butterfly and benes networks in [26]. For detailed results on the topological indices of graphs the readers may refer to $[1-6,8,12,17,18,37,38]$.

The following presents an example of how the indices can be used in chemical engineering.

Example. The generalized first Zagerb index is formulated as $\sum_{v \in V(G)} d_{v}^{\alpha}$ where $\alpha$ is a real number. When $\alpha=2$ it becomes an equation (4), and when $\alpha=3$ it becomes a forgotten index which is stated as

$$
F(G)=\sum_{v \in V(G)} d_{v}^{3}=\sum_{u v \in E(G)}\left(d_{u}^{2}+d_{v}^{2}\right)
$$

As we can see in website http://www.moleculardescriptors. $\mathrm{eu} /$ dataset/dataset.htm, in accordance to the International Academy of Mathematical Chemistry, the potential ability of this index was tested in terms of a data set of octane isomers. In the simplest form, the $F$ index doesn't identify multiple bonds and hetero atoms, which becomes the reason why a data set is selected as a measure. A list of data including entropy, heat of vaporization, boiling point, density, heat capacities, melting point, enthalpy of formation, motor octane number, acentric factor, octanol-water partition coefficient, molar refraction, total surface area, and molar volume help to compose the octane data set. The $F$ index indicates its strong bonds with most characteristics. As a result, the $F$ index is verified to have correlation coefficients larger than 0.95 in the entropy and acentric factor.

For various other chemical features, whereas, $F$ index may not be strongly related. To strengthen the predictive ability of the $F$ index in possible chemical application circumstances, a linear framework was introduced as follows (see Furtula and Gutman [20]):

$$
\sum_{u v \in E(G)}\left(d_{u}+d_{v}\right)+\lambda \sum_{u v \in E(G)}\left(d_{u}^{2}+d_{v}^{2}\right),
$$


where $\lambda$ is a balanced parameter adjusted to the specific applications in chemical engineering (generally speaking, $\lambda$ always takes a value in interval $[-20,20])$; the first term $\sum_{u v \in E(G)}\left(d_{u}+d_{v}\right)$ is the first Zagreb index described in (4). In light of massive experimental studies, this framework can be applied in every chemical property with a certain octane database. For instance, a significant improvement can be yielded in the octanol-water partition coefficient, and it's revealed that the absolute value of the correlation coefficient infers a sharp maximum by taking $\lambda=-0.14$ in the above computing model. $\log P$ is the $\log$ arithm function of the octanol-water partition coefficient. Hence, by means of derivation, the octanol-water partition coefficient of octanes can be formulated as follows:

$$
\begin{aligned}
\log P & =-0.2058\left(\sum_{u v \in E(G)}\left(d_{u}+d_{v}\right)\right. \\
& \left.-0.14 \sum_{u v \in E(G)}\left(d_{u}^{2}+d_{v}^{2}\right)\right)+7.5864 .
\end{aligned}
$$

This fact indicates that the correlation coefficient can reach 0.99896 and the mean absolute percentage error is only $0.06 \%$.

The paper is structured below: In Section 2, we compute the Randić, first and second Zagreb , hyper Zagreb, first and second multiple Zagreb, general sum connectivity, $A B C, A B C_{4}, G A$ and $G A_{5}$ indices for OTIS swapped networks by taking path and $k$-regular graphs on $n$ vertices as a base graph. In Section 3, we give explicit formulas of these indices for the OTIS biswapped networks by considering a basis graph as a path and $k$-regular graph of order $n$.

\section{Topological indices of OTIS swapped networks}

Definition 2.1. The OTIS swapped network $O_{\Omega}$, deduced from the graph $\Omega$, is a graph with vertex set $V\left(O_{\Omega}\right)=\{\langle g, p\rangle \mid g, p \in V(\Omega)\}$ and edge set $E\left(O_{\Omega}\right)=$ $\left\{\left(\left\langle g, p_{1}\right\rangle,\left\langle g, p_{2}\right\rangle\right) \mid g \in V(\Omega),\left(p_{1}, p_{2}\right) \in E(\Omega)\right\} \cup$ $\{(\langle g, p\rangle,\langle p, g\rangle) \mid g, p \in V(\Omega)$ and $g \neq p\}$.

In OTIS swapped network $O_{\Omega}$, the graph $\Omega$ is named the basis (factor) graph or network. If the basis network $\Omega$ has $n$ nodes, then $O_{\Omega}$ is composed of $n$ node-disjoint subnetworks called clusters, which are isomorphic to $\Omega$. The node label $\langle g, p\rangle$ in $O_{\Omega}$ define the node indexed $p$ in cluster $g$. The vertex and edge set cardinalities of OTIS Swapped network $O_{\Omega}$ are $n^{2}$ and $n|E(\Omega)|+\frac{n(n-1)}{2}$ respectively, where $n$ is the number of vertices in $\Omega$. Figure 1 depicts an OTIS swapped network with a complete graph of order $6\left(K_{6}\right)$ as the basis graph.

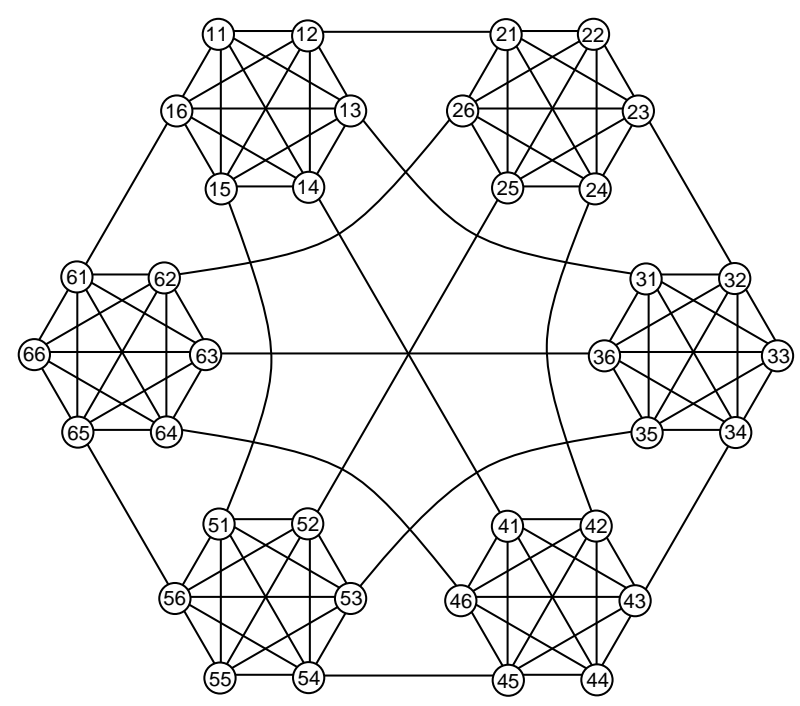

Figure 1: OTIS swapped network $O_{K_{6}}$

\subsection{Results for OTIS Swapped networks $\boldsymbol{O}_{P_{n}}$}

Set $P_{n}$ as a path on $n$ vertices and $O_{P_{n}}$ as OTIS swapped network with basis network $P_{n}$. An OTIS swapped network with the basis network $P_{6}$ is shown in Figure 2 .

Table 1: The edge partition of the graph $O_{P_{n}}$

\begin{tabular}{ccccc}
\hline $\begin{array}{c}\left(d_{u}, d_{v}\right) \text { where } \\
u v \in E(G)\end{array}$ & $(1,3)$ & $(2,2)$ & $(2,3)$ & $(3,3)$ \\
$\begin{array}{c}\text { Number of edges } \\
\text { umber }\end{array}$ & 2 & 3 & $6 n-14$ & $\frac{3(n-2)(n-3)}{2}$ \\
\hline
\end{tabular}

Now the certain degree based topological indices of OTIS swapped network $O_{P_{n}}$ is computed.

Theorem 2.2. The general Randic index and the general sum connectivity index of $O_{P_{n}}$ is equal to

$$
\begin{aligned}
R_{\alpha}\left(O_{P_{n}}\right) & =2 \times 3^{\alpha}+3 \times 4^{\alpha}+(6 n-14) \times 6^{\alpha} \\
& +\frac{3(n-2)(n-3)}{2} \times 9^{\alpha},
\end{aligned}
$$

$\chi_{\alpha}\left(O_{P_{n}}\right)=5 \times 4^{\alpha}+(6 n-14) \times 5^{\alpha}+\frac{3(n-2)(n-3)}{2} \times 6^{\alpha}$.

Proof. The number of vertices and edges in $O_{P_{n}}$ are $n^{2}$ and $\frac{3 n(n-1)}{2}$ respectively. There are four types of edges in 


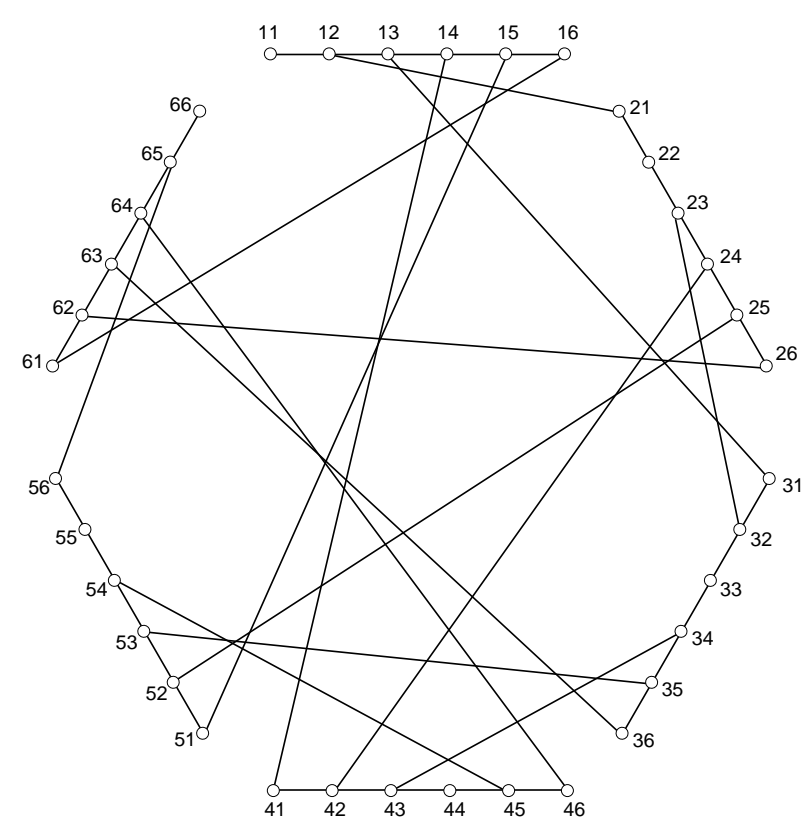

Figure 2: OTIS swapped network $O_{P_{6}}$

$O_{P_{n}}$ based on degrees of end vertices of each edge. Table 1 shows such an edge partition for $O_{P_{n}}$. Using formula (1) and (3) in Table 1, we obtain the expression of the indices.

From the above theorem, one can immediately compute Randić connectivity, first and second Zagreb, hyper Zagreb and sum connectivity indices of OTIS swapped network $O_{P_{n}}$.

Corollary 2.3. Let $G$ be the graph of OTIS swapped network $O_{P_{n}}$. Then

$$
\begin{gathered}
R_{\frac{-1}{2}}(G)=\frac{n^{2}}{2}+\left(\sqrt{6}-\frac{5}{2}\right) n+\frac{2}{\sqrt{3}}-\frac{14}{\sqrt{6}}+\frac{9}{2}, \\
M_{1}(G)=9 n^{2}-15 n+4, \\
M_{2}(G)=\frac{27 n^{2}-63 n+30}{2}, \\
H M(G)=54 n^{2}-120 n+106, \\
\operatorname{SCI}(G)=\frac{3 n^{2}-15 n+18+5 \sqrt{6}}{2 \sqrt{6}}+\frac{6 n-14}{\sqrt{5}} .
\end{gathered}
$$

In the theorem below, we calculate first and second multiple Zagreb, atom bond connectivity and geometric arithmetic indices of the OTIS swapped network $O_{P_{n}}$.

Theorem 2.4. Consider an OTIS swapped network $O_{P_{n}}$, then

$$
P M_{1}\left(O_{P_{n}}\right)=8640\left(3 n^{3}-22 n+53 n-42\right),
$$

$$
\begin{gathered}
P M_{2}\left(O_{P_{n}}\right)=11664\left(3 n^{3}-22 n+53 n-42\right), \\
A B C\left(O_{P_{n}}\right)=n^{2}+n(\sqrt{18}-5)+2 \sqrt{\frac{2}{3}}+\frac{3 \sqrt{2}}{2}+6-14 \sqrt{\frac{3}{6}}, \\
G A\left(O_{P_{n}}\right)=\frac{3 n^{2}}{2}+n\left(\frac{12 \sqrt{6}}{5}-\frac{15}{2}\right)+9+\sqrt{3}-\frac{28 \sqrt{6}}{5} .
\end{gathered}
$$

Proof. The edge partition based on the degree of end vertices of each edge is shown in the Table 1. We apply formulas (6), (7), (9) and (11) to the information in Table 1 and obtain the required results.

\subsection{Results for OTIS (Swapped) networks $\boldsymbol{O}_{R_{k}}$}

Let $R_{k}$ be $k$-regular graph on $n$ vertices and $O_{R_{k}}$ be the OTIS swapped network with the basis network $R_{k}$. Figure 1 depicts an example of OTIS swapped network $O_{K_{6}}$. Now we calculate certain degree based topological indices of the OTIS swapped network $O_{R_{k}}$.

Theorem 2.5. Let $O_{R_{k}}$ be an OTIS swapped network, then its general Randic and the general sum connectivity index is equal to

$$
\begin{aligned}
& R_{\alpha}\left(O_{R_{k}}\right)=n k\left(k^{2}+k\right)^{\alpha}+\frac{n^{2}(k+1)-n(2 k+1)}{2}(k+1)^{2 \alpha}, \\
& \chi_{\alpha}\left(O_{R_{k}}\right)=n k(2 k+1)^{\alpha}+\frac{n^{2}(k+1)-n(2 k+1)}{2}(2 k+2)^{\alpha} .
\end{aligned}
$$

Proof. The number of vertices and edges in $O_{R_{k}}$ are $n^{2}$ and $\frac{n^{2}(k+1)-n}{2}$ respectively. Two sorts of edges exist in $O_{R_{k}}$ which are based on degrees of the end vertices of each edge. Such an edge partition of $O_{R_{k}}$ can be found in Table 2. Using formula (1) and (3) in Table 1, we obtain the expression of the indices.

Table 2: The edge partition of the graph $O_{R_{k}}$

\begin{tabular}{ccc}
\hline $\begin{array}{c}\left(d_{u}, d_{v}\right) \text { where } \\
u v \in E(G)\end{array}$ & $(k, k+1)$ & $(k+1, k+1)$ \\
Number of edges & $n k$ & $\frac{n^{2}(k+1)-n(1+2 k)}{2}$ \\
\hline
\end{tabular}

From the above theorem, one can compute Randić connectivity, first and second Zagreb, hyper Zagreb and sum connectivity indices of OTIS swapped network $O_{R_{k}}$.

Corollary 2.6. Let $G$ be the graph of OTIS swapped network $O_{R_{k}}$. Then

$$
R_{\frac{-1}{2}}(G)=\frac{n k}{\sqrt{k^{2}+k}}+\frac{n^{2}(k+1)-n(2 k+1)}{2 k+2},
$$




$$
\begin{gathered}
M_{1}(G)=n^{2}(k+1)^{2}-n(2 k+1), \\
M_{2}(G)=\frac{n^{2}(k+1)^{3}-n(k+1)(3 k-1)}{2}, \\
H M(G)=2 n^{2}(k+1)^{3}-n(2 k+1)(3 k+2), \\
S C I(G)=\frac{n k}{\sqrt{2 k+1}}+\frac{n^{2}(k+1)-n(2 k+1)}{2 \sqrt{2 k+2}} .
\end{gathered}
$$

In the theorem below, we calculate first and second multiple Zagreb, atom bond connectivity and geometric arithmetic indices of the OTIS swapped network $O_{R_{k}}$.

Theorem 2.7. Consider an OTIS swapped network $O_{R_{k}}$, then

$$
\begin{gathered}
P M_{1}\left(O_{R_{k}}\right)=n k(k+1)(2 k+1)\left(n^{2}(k+1)-n(2 k+1)\right), \\
P M_{2}\left(O_{R_{k}}\right)=\frac{n k^{2}(k+1)^{3}\left(n^{2}(k+1)-n(2 k+1)\right)}{2}, \\
A B C\left(O_{R_{k}}\right)=\frac{\sqrt{2 k} n^{2}}{2}+n\left(k \sqrt{\frac{2 k-1}{k^{2}+k}}-\frac{\sqrt{2 k}(2 k+1)}{2(k+1)}\right), \\
G A\left(O_{R_{k}}\right)=\frac{n^{2}(k+1)}{2}+n\left(\frac{2 k \sqrt{k^{2}+k}}{2 k+1}-\frac{2 k+1}{2}\right) .
\end{gathered}
$$

Proof. The edge partition based on the degree of end vertices of each edge is described in the Table 2 . We practice formulas (6), (7), (9) and (11) to the information in Table 2 and obtain the expected results.

\section{Topological indices of Biswapped networks}

For a base graph $\Omega$, the biswapped interconnection network $B s w(\Omega)$ is a graph with vertex set and edge set specified as:

$$
\begin{gathered}
V(B s w(\Omega))=\{\langle 0, p, g\rangle,\langle 1, p, g\rangle \mid p, g \in V(\Omega)\} \\
E(B s w(\Omega))=\left\{\left(\left\langle 0, p, g_{1}\right\rangle,\left\langle 0, p, g_{2}\right\rangle\right),\left(\left\langle 1, p, g_{1}\right\rangle,\right.\right. \\
\left.\left.\left\langle 1, p, g_{2}\right\rangle\right) \mid\left(g_{1}, g_{2}\right) \in E(\Omega), p \in V(\Omega)\right\} \\
\cup\{(\langle 0, p, g\rangle,\langle 1, g, p\rangle) \mid p, g \in V(\Omega)\}
\end{gathered}
$$

The definition postulate $2 n$ clusters, whereby each cluster is a $\Omega$ graph. The $n$ clusters with nodes labelled $\langle 0$, cluster $\sharp$, node $\sharp\rangle$ form part 0 of the bipartite graph and the remaining $n$ cluster constitutes part 1 with associated nodes numbers $\langle 1$, cluster $\sharp$, node $\sharp\rangle$. Each cluster $p$ in either part of $B s w(\Omega)$ has the similar internal connectivity as $\Omega$. Moreover, node $g$ of cluster $p$ in part 0 is connected to node $p$ of cluster $g$ of part 1 .

As an example, when $\Omega=C_{4}$ constitutes the basis graph then Figure 3 represent the resulting $B s w(\Omega)$. Part 0 of the network is drawn at the top and part 1 at the bottom, with cluster $0-3$ positioned from left to right. The vertex and edge set cardinalities of biswapped network $B s w(\Omega)$ are $2 n^{2}$ and $2 n|E(\Omega)|+n^{2}$ respectively, where $n$ is the number of vertices in $\Omega$. Some topological properties of the biswapped network are studied in [36].

Now we calculate certain degree based topological indices of the biswapped network.

\subsection{Results for Biswapped networks $\operatorname{Bsw}\left(P_{n}\right)$}

Let $P_{n}$ be path on the $n$ vertices and $B s w\left(P_{n}\right)$ be the biswapped network with the basis network $P_{n}$. The number of vertices and edges in $B s w\left(P_{n}\right)$ are $2 n^{2}$ and $3 n^{2}-2 n$ respectively. Figure 4 shows a biswapped network with the 5-node path $P_{5}$ as the basis graphs. We compute the general Randić and the general sum connectivity index in the theorem below.

Theorem 3.1. Let Bsw $\left(P_{n}\right)$ be a biswapped network, then its general Randic and general sum connectivity index equals to

$$
\begin{aligned}
& R_{\alpha}\left(B s w\left(P_{n}\right)\right)=4 \times 4^{\alpha}+8(n-1) 6^{\alpha}+\left(3 n^{2}-10 n+4\right) 9^{\alpha}, \\
& \chi_{\alpha}\left(B s w\left(P_{n}\right)\right)=4 \times 4^{\alpha}+8(n-1) 5^{\alpha}+\left(3 n^{2}-10 n+4\right) 6^{\alpha} .
\end{aligned}
$$

Proof. The number of vertices and edges in $B s w\left(P_{n}\right)$ are $2 n^{2}$ and $3 n^{2}-2 n$ respectively. There are three types of edges in $B s w\left(P_{n}\right)$ based on degrees of end vertices of each edge. Table 3 reveals such an edge partition of $B s w\left(P_{n}\right)$. Using formulas (1) and (3) in table 3 , we obtain the expression of the indices. $\quad \square$ From the above theorem, one can

Table 3: The edge partition of the graph $B s w\left(P_{n}\right)$

\begin{tabular}{lccc}
\hline $\begin{array}{c}\left(d_{u}, d_{v}\right) \text { where } \\
u v \in E\left(B s w\left(P_{n}\right)\right)\end{array}$ & $(2,2)$ & $(2,3)$ & $(3,3)$ \\
Number of edges & 4 & $8(n-1)$ & $3 n^{2}-10 n+4$ \\
\hline
\end{tabular}




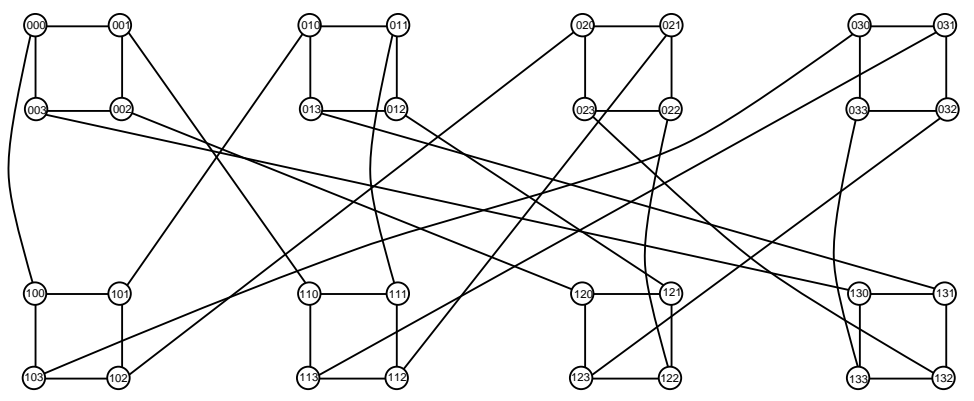

Figure 3: Biswapped network $B s w\left(C_{4}\right)$

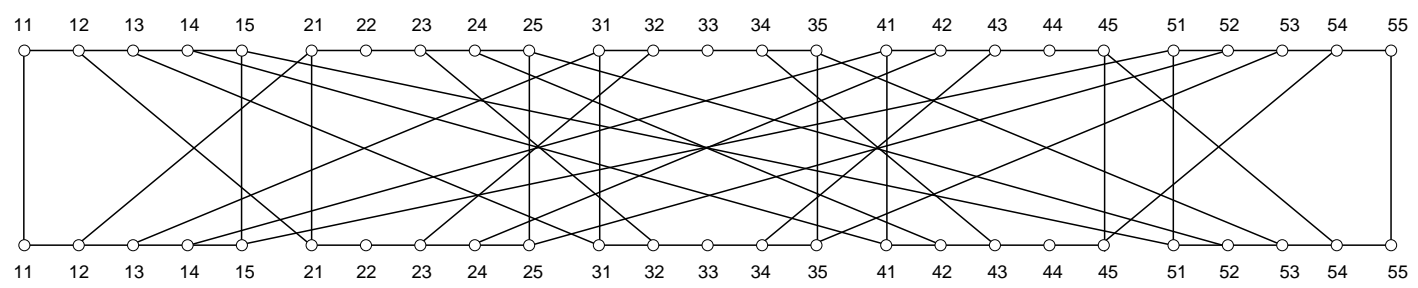

Figure 4: Biswapped network $B s w\left(P_{5}\right)$

compute Randić connectivity, first and second Zagreb, hyper Zagreb and sum connectivity indices of OTIS swapped network $B s w\left(P_{n}\right)$.

Corollary 3.2. Let $G$ be the graph of OTIS swapped network $\operatorname{Bsw}\left(P_{n}\right)$. Then

$$
\begin{gathered}
R_{\frac{-1}{2}}(G)=n^{2}+\left(\frac{4}{3} \sqrt{6}-\frac{10}{3}\right) n+\frac{10}{3}-\frac{4}{3} \sqrt{6}, \\
M_{1}(G)=18 n^{2}-20 n, \\
M_{2}(G)=27 n^{2}-42 n+4, \\
H M(G)=108 n^{2}-160 n+8, \\
\operatorname{SCI}(G)=\frac{3 n^{2}}{\sqrt{6}}+n\left(\frac{8}{\sqrt{5}}-\frac{10}{\sqrt{6}}\right)+2+\frac{4}{\sqrt{6}}-\frac{1}{\sqrt{5}} .
\end{gathered}
$$

In the theorem below, we calculate atom bond connectivity, first and second multiple Zagreb, and geometric arithmetic indices of the OTIS swapped network $B s w\left(P_{n}\right)$.

Theorem 3.3. Consider an OTIS swapped network $\operatorname{Bsw}\left(P_{n}\right)$, then

$$
\begin{aligned}
& P M_{1}\left(B s w\left(P_{n}\right)\right)=3840\left(3 n^{3}-13 n^{2}+14 n-4\right), \\
& P M_{2}\left(B s w\left(P_{n}\right)\right)=6912\left(3 n^{3}-13 n^{2}+14 n-4\right),
\end{aligned}
$$

$$
\begin{aligned}
& A B C\left(B s w\left(P_{n}\right)\right)=2 n^{2}+\left(4 \sqrt{2}-\frac{20}{3}\right) n-2 \sqrt{2}+\frac{8}{3}, \\
& G A\left(B s w\left(P_{n}\right)\right)=3 n^{2}+\left(\frac{16}{5} \sqrt{6}-10\right) n+8-\frac{16}{5} \sqrt{6} .
\end{aligned}
$$

Proof. The edge partition based on the degree of end vertices of each edge is described in the Table 3 . We practice formulas (6), (7), (9) and (11) to the information in Table 3 and obtain the expected results.

Theorem 3.4. Consider $n \geq 5$ and $B s w\left(P_{n}\right)$ a biswapped network with basis network $P_{n}$, then

$$
\begin{aligned}
& A B C_{4}\left(B s w\left(P_{n}\right)\right) \\
& =\frac{4}{3} n^{2}+\left(-\frac{53}{9}+\frac{\sqrt{462}}{21}+\frac{3}{4} \sqrt{14}+\frac{1}{3} \sqrt{30}\right) n \\
& +\frac{8}{5} \sqrt{2}-\frac{13}{7} \sqrt{14}+\frac{40}{9}+\frac{2}{7} \sqrt{182}-\frac{2}{3} \sqrt{30} . \\
& G A_{5}\left(B s w\left(P_{n}\right)\right) \\
& =3 n^{2}+\left(\frac{4}{13} \sqrt{42}+\frac{24}{7} \sqrt{3}-14+\frac{48}{17} \sqrt{2}\right) n \\
& +8+\frac{4}{3} \sqrt{35}-\frac{64}{7} \sqrt{3}+\frac{32}{15} \sqrt{14}-\frac{96}{17} \sqrt{2} .
\end{aligned}
$$

Proof. Let $B s w\left(P_{n}\right)$ be a biswapped network with basis network $P_{n}$. We prove it by considering the edge partition in Table 4. We practice formulas (10) and (12) to the information provided in Table 4 and obtain the expected results. 
Table 4: The edge partition of the graph $B s w\left(P_{n}\right)$

\begin{tabular}{cccc}
\hline $\begin{array}{c}\left(S_{u}, S_{v}\right) \\
\text { where } \\
u v \in E(G)\end{array}$ & $\begin{array}{c}\text { Number of } \\
\text { edges }\end{array}$ & $\begin{array}{c}\left(S_{u}, S_{v}\right) \\
\text { where } \\
u v \in E(G)\end{array}$ & $\begin{array}{c}\text { Number of } \\
\text { edges }\end{array}$ \\
\hline$(5,5)$ & 4 & $(5,7)$ & 8 \\
$(6,7)$ & $2 n$ & $(6,8)$ & $6 n-16$ \\
$(7,8)$ & 8 & $(8,8)$ & $6 n-24$ \\
$(8,9)$ & $4 n-8$ & $(9,9)$ & $3 n^{2}-20 n+$ \\
& & & 28 \\
\hline
\end{tabular}

\subsection{Results for Biswapped networks $\operatorname{Bsw}\left(R_{k}\right)$}

Let $R_{k}$ be $k$-regular graph of order $n$ and let $B s w\left(R_{k}\right)$ be the biswapped network with the basis network $R_{k}$. We can see that $B s w\left(R_{k}\right)$ is a $k+1$ regular graph of order $2 n$ and size $n^{2}(k+1)$. A biswapped network with the basis network $C_{4}$ is shown in Figure 3. We compute the general Randic and the general sum connectivity index in the following theorem.

Theorem 3.5. Let $B s w\left(R_{k}\right)$ be a biswapped network, then its general Randic and general sum connectivity index is equal to

$$
\begin{gathered}
R_{\alpha}\left(B s w\left(R_{k}\right)\right)=n^{2}(k+1)^{2 \alpha+1}, \\
\chi_{\alpha}\left(B s w\left(R_{k}\right)\right)=2^{\alpha} n^{2}(k+1)^{\alpha+1} .
\end{gathered}
$$

Proof. The number of vertices and edges in $B s w\left(R_{k}\right)$ are $2 n$ and $n^{2}(k+1)$ respectively. In the biswapped network $B s w\left(R_{k}\right), \operatorname{deg}(u)=k+1$ for every vertex $u$ in $B s w\left(R_{k}\right)$. Using formulas (1) and (3), we obtain the expression of the indices.

The Randić connectivity, first and second Zagreb, first and second multiple Zagreb, hyper Zagreb and sum connectivity indices of OTIS swapped network $B s w\left(R_{k}\right)$ is sated in the following corollary.

Corollary 3.6. Let $G$ be the graph of the OTIS swapped network Bsw $\left(R_{k}\right)$. Then

$$
\begin{gathered}
R_{\frac{-1}{2}}(G)=n^{2}, \\
P M_{1}(G)=M_{1}(G)=2 n^{2}(k+1)^{2}, \\
P M_{2}(G)=M_{2}(G)=n^{2}(k+1)^{3}, \\
H M(G)=4 n^{2}(k+1)^{3},
\end{gathered}
$$

$$
\operatorname{SCI}(G)=\frac{n^{2}(k+1)}{\sqrt{2 k+2}} .
$$

Next, the atom-bond connectivity index and fourth version of atom-bond connectivity index of Biswapped network $\operatorname{Bsw}\left(R_{k}\right)$ is caculated.

Theorem 3.7. Let $B s w\left(R_{k}\right)$ be a biswapped network, then

$$
A B C\left(B s w\left(R_{k}\right)\right)=n^{2} \sqrt{2 k},
$$

$$
A B C_{4}\left(B s w\left(R_{k}\right)\right)=\frac{n^{2} \sqrt{2 k^{2}+4 k}}{k+1} .
$$

Proof. The biswapped network is a $k+1$ regular graph i.e., the set of neighbors is the same for every vertex. The sum of degrees of all vertices adjacent to every vertex $u$ is $(k+$ $1)^{2}$. Thus $\sqrt{\frac{d_{u}+d_{v}-2}{d_{u} d_{v}}}=\frac{\sqrt{2 k}}{k+1}$ and $\sqrt{\frac{S_{u}+S_{v}-2}{S_{u} S_{v}}}=\frac{\sqrt{2 k^{2}+4 k}}{(k+1)^{2}}$ for every edge $u v \in B s w\left(R_{k}\right)$. Then according to formula (9), we have

$$
\begin{aligned}
& A B C\left(B s w\left(R_{k}\right)\right) \\
& =\sum_{u v \in E\left(B s w\left(R_{k}\right)\right)} \sqrt{\frac{d_{u}+d_{v}-2}{d_{u} d_{v}}} \\
& =n^{2}(k+1) \cdot \frac{\sqrt{2 k}}{k+1}=n^{2} \sqrt{2 k}
\end{aligned}
$$

and according to formula (10) we obtain

$$
\begin{aligned}
& A B C_{4}\left(\operatorname{Bsw}\left(R_{k}\right)\right) \\
& =\sum_{u v \in E\left(B s w\left(R_{k}\right)\right)} \sqrt{\frac{S_{u}+S_{v}-2}{S_{u} S_{v}}} \\
& =n^{2}(k+1) \cdot \frac{\sqrt{2 k^{2}+4 k}}{(k+1)^{2}}=\frac{n^{2} \sqrt{2 k^{2}+4 k}}{k+1} .
\end{aligned}
$$

Hence, we finish the proof.

In the theorem below, we determine the geometricarithmetic $G A$ index and fifth version of the $G A$ index of the biswapped network.

Theorem 3.8. Consider a Biswapped network $B s w\left(R_{k}\right)$, then

$$
\begin{gathered}
G A\left(B s w\left(R_{k}\right)\right)=n^{2}(k+1), \\
G A_{5}\left(B s w\left(R_{k}\right)\right)=n^{2}(k+1)^{2} .
\end{gathered}
$$

Proof. It is easy to see that $\frac{2 \sqrt{d_{u} d_{v}}}{d_{u}+d_{v}}=1$ and $\frac{2 \sqrt{S_{u} S_{v}}}{S_{u}+S_{v}}=k+1$ for every edge $u v \in B s w\left(R_{k}\right)$. Then using formula (11) for the geometric-arithmetic index we have

$$
G A\left(B s w\left(R_{k}\right)\right)=\sum_{u v \in E\left(B s w\left(R_{k}\right)\right)} \frac{2 \sqrt{d_{u} d_{v}}}{d_{u}+d_{v}}=n^{2}(k+1) .
$$


According to Formula (12) for the fifth version of geometricarithmetic index we obtain

$$
G A_{5}\left(B s w\left(R_{k}\right)\right)=\sum_{u v \in E\left(B s w\left(R_{k}\right)\right)} \frac{2 \sqrt{S_{u} S_{v}}}{S_{u}+S_{v}}=n^{2}(k+1)^{2} .
$$

The desired result is proved.

\section{Conclusion}

This paper addressed the OTIS swapped networks and biswapped networks and researched on their topological indices. We determined the general Randić, general sum connectivity, first and second Zagreb, first and second multiple Zagreb, hyper Zagreb atombond and geometric indices for both the family of networks by considering the basis network as path $P_{n}$ and $k$-regular graph $R_{k}$. We also gave explicit formulae for $A B C_{4}$ and $G A_{5}$ indices of these networks with the basis network $R_{k}$.

Acknowledgement: This work has been partially supported by National Science Foundation of China (no. 11761083).

\section{References}

[1] Aslam A., Ahmed S., Gao W., On topological indices of boron triangular nanotubes, Z. Naturforsch., 2017, 72, 711-716.

[2] Aslam A., Bashir Y., Ahmed S., Gao W., On topological indices of certain dendrimer structures, Z. Naturforsch., 2017, 72, 559-566.

[3] Aslam A., Jamil M.K., Gao W., Nazeer W., On topological ascpects of some dendrimer structures, Nanotechnology Reviews., 2018, 7, 123-129.

[4] Baig A.Q., Naeem M., Revan and hyper revan indices of octahedral and icosahedral networks, App. Math. Nonl. Sci., 2018, 3 , 33-40.

[5] Bashir Y., Aslam A., Kamran M., et al., On forgotten topological indices of some dendrimers structure, Molecules, 2017, 22, doi: 10.3390/molecules22060867.

[6] Aslam A., Guirao J.L.G., Ahmad S., Gao W., Topological indices of the line graph of subdivision graph of complete bipartite graphs, Appl. Math. Inf. Sci., 2017, 11, 1631-1636.

[7] Al-Ayyoub A., Awwad A., Day K., Ould-Khaoua M., Generalized methods for algorithm development on optical systems, J. Supercomputing., 2006, 38, 111-125.

[8] Baca M., J. Horváthová, M. Mokrišová, et al. On topological indices of fullerenes, Appl. Math. Comput., 2015, 251, 154-161.

[9] Bollobas B., Erdos P., Graphs of extremal weights, Ars Combin., 1998, 50, 225-233.

[10] Zhou B., Trinajstić N., On a novel connectivity index, J. Math. Chem., 2009, 46, 1252-1270.

[11] Zhou B., Trinajstić N., On general sum-connectivity index, J. Math. Chem., 2010, 47, 210-218.
[12] Chen J., Liu J., Guo X., Some upper bounds for the atom-bond connectivity index of graphs, Appl. Math. Lett., 2012, 25, 10771081.

[13] Wang C.F., Sahni S., Basic operations on the OTIS-Mesh optoelectronic computer, IEEE T. Parall. Distr. Syst., 1998, 9, 1226-1236.

[14] Wang C.F., Sahni S., Image processing on the OTIS-Mesh optoelectronic computer, IEEE T. Parall. Distr. Syst., 2000, 11, 97-109.

[15] Wang C.F., Sahni S., Matrix multiplication on the OTISMesh optoelectronic computer, IEEE T. Comput., 2001, 50, 635-646.

[16] Wang C.F., Sahni S., OTIS optoelectronic computers, Chapter 5 in Parallel Computation Using Optical Interconnection, K. Li, Y. Pan, and S.Q. Zhang, eds., Kluwer Academic Publishers, 1998, 99-116.

[17] Das K.C., Gutman I., Furtula B., On atom-bond connectivity index, Chem. Phys. Lett., 2011, 511, 452-454.

[18] Du Z., Zhou B., Trinajstić N., Minimum general sum-connectivity index of unicyclic graphs, J. Math. Chem., 2010, 48, 697-703.

[19] Estrada E., Torres L., Rodriguez L., et al. An atom-bond connectivity index: Modelling the enthalpy of formation of alkanes, Indian J. Chem., 1998, 37, 849-855.

[20] Furtula B., Gutman I., A forgotten topological index, J. Math. Chem., 2015, 53, 1184-1190.

[21] Ghorbani M., Azimi N., Note on multiple Zagreb indices, Iran. J. Math. Chem., 2012, 3, 137-143.

[22] Ghorbani M., Hosseinzadeh M.A., Computing $A B C_{4}$ index of nanostar dendrimers, optoelectron, Adv. Mater. Rapid Commun., 2010, 4, 1419-1422.

[23] Graovac A., Ghorbani M., Hosseinzadeh M.A., Computing fifth geometric-arithmetic index for nanostar dendrimers, J. Math. Nanosci, 2011, 1, 33-42.

[24] Marsden G., Marchand P., Harvey P., Esener S., Optical transpose interconnection system architecture, Opt. Lett., 1993, 18, 10831085.

[25] Hayat S., Imran M., Computation of topological indices of certain networks, Appl. Math. Comput., 2014, 240, 213-228.

[26] Hayat S., Imran M., Mailk M.Y.H., On topological indices of certain interconnection networks, Appl. Math. Comput., 2014, 244, 936951.

[27] Day K., Optical transpose $k$-ary $n$-cube networks, J. Systems Architecture, 2004, 50, 697-705.

[28] Day K., Al-Ayyoub A., Topological properties of OTIS-networks, IEEE T. Parall. Distr. Syst., 2002, 14, 359-366.

[29] Jana P.K., Polynomial interpolation and polynomial root finding on OTIS-mesh, Parall. Comput., 2006, 32, 301-312.

[30] Jana P.K., Sinha B.P., An improved parallel prefix algorithm on OTIS-mesh, Parall. Proc. Leters. 2006, 16, 429-440.

[31] Rajasekaran S., Sahni S., Randomized routing, selection, and sorting on the OTIS-mesh, IEEE T. Parall. Distr. Syst., 1998, 9, 833-840.

[32] Randić M., On characterization of molecular branching, J. Am. Chem. Soc., 1975, 97, 6609-6615.

[33] Shirdel G.H., Pour H.R., Sayadi A.M., The hyper-Zagreb index of graph operations, Iran. J. Math. Chem., 2013, 4, 213-220.

[34] Vukičević D., Furtula B., Topological index based on the ratios of geometrical and arithmetical means of end-vertex degrees of edges, J. Math. Chem., 2009, 46, 1369-1376.

[35] Wiener H.J., Structural determination of paraffin boiling points, J. Amer. Chem. Soc., 1947, 69, 17-20. 
[36] Xiou W., Chen W., He M., Wei W., Parhami B., Biswapped networks and their topological properties, ACIS International conference on software engineering, Artificial Intelligence, Networking and Paralell Distributed computing (SNPD) IEEE, 2007, 2, 193-198.

[37] Bashir Y., Aslam A., Kamran M., et al. On forgotten topological indices of some dendrimer structures, Molecules, 2017, 22, doi:10.3390/molecules22060867.

[38] Zhao B., Gan J., Wu H., Redefined Zagreb indices of some nanostructures, App. Math. Nonl. Sci., 2016, 1, 291-300. 